050

VALUTAZIONE DELL'ATTIVITÀ ANTI-BATTERICA “IN VITRO”

DELL'ULIFLOXACINA NEI CONFRONTI DEI PRINCIPALI BATTERI GRAM NEGATIVI UROPATOGENI

Botta F., Arighi E., Casartelli R., Moretti G., Pieretti B., Tettamanti B., Terramocci R.

Laboratorio Analisi Ospedale "Valduce", via Dante 1 1, 22100 Como (CO)

Introduzione. Scopo di questo studio è stato quello di valutare “in vitro” l'efficacia dell’Ulifloxacina (ULI), un chinolonico di nuova generazione, su batteri Gram negativi causa di infezioni delle vie urinarie, comparandolo con i chinolonici già in uso. Studi clinici hanno dimostrato che tale farmaco risulta essere molto efficace nel trattamento delle infezioni uroginecologiche, in quanto raggiunge un'elevata concentrazione nei tessuti del tratto urogenitale femminile, oltre che nelle urine, mantenendo a differenza degli altri chinolonici di uso comune inalterata la fisiologica flora lattobacillare.

Materiali e Metodi. Sono stati testati 184 campioni di urine positivi per batteri Gram negativi con metodica Kirby-Bauer (inoculo 0.5 McFarland su terreno Mueller Hinton incubato a $37^{\circ} \mathrm{C}$ per $24 \mathrm{~h}$ ) con i seguenti antibioti-

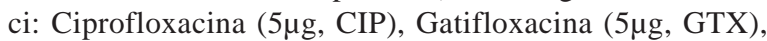

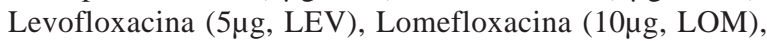

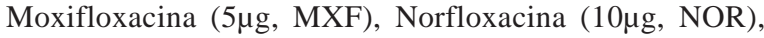
Ofloxacina $(5 \mu \mathrm{g}, \mathrm{OFX})$, Ulifloxacina $(5 \mu \mathrm{g}$, ULI). Gli isolati batterici indagati sono rappresentati da: Escherichia coli (47,8\%), Enterobacter aerogenes (3,3\%), Enterobacter cloacae (3,8\%), Klebsiella oxytoca (3,8\%), Klebsiella pneumoniae (13,6\%), Proteus mirabilis (15,2\%), Pseudomonas aeruginosa (8,7\%), altri germi Gram negativi (3,8\%).

Risultati e Discussione. Dall'analisi dei dati sono risultati: resistenti a tutti i chinolonici testati il $20.5 \%$ di E coli, $50 \%$ di E. aerogenes e P. aeruginosa, $71.4 \%$ di E. cloacae, 8\% di K. pneumoniae e $37 \%$ di P. mirabilis; sensibili a tutti i chinolonici testati tranne alla MXF il $68.2 \%$ di E. coli, $33.3 \%$ di E. aerogenes, $28.6 \%$ di E. cloacae, $85.7 \%$ di $K$. oxytoca, $84 \%$ di K. pneumoniae e $44.4 \%$ di P. mirabilis; casi discordanti (ULI intermedia o sensibile rispetto agli altri chinolonici) per E. coli (2.3\%), E. aerogenes (16.7\%), K. Pneumoniae (4\%), P. mirabilis (7.4\%) e P. aeruginosa (6.3\%).

Per quanto riguarda quest'ultimo punto l'ULI si è dimostrata l'unica a sensibilità intermedia contro la resistenza registrata per gli altri chinolonici nel $2.3 \%$ dei casi di E.coli e nel $16.7 \%$ di E.aerogenes, mentre è risultata l'unica sensibile a fronte di resistenza più o meno accentuata degli altri antibiotici nel 4\% dei casi di K.pneumoniae, nel $7.4 \%$ di P.mirabilis e nel $6.3 \%$ di P.aeruginosa.

Conclusioni. In aggiunta ai casi discussi l'ULI si è rivelata efficace "in vitro" tanto quanto gli altri chinolonici, in accordo con i dati riportati in letteratura, ed inoltre è possibile suggerirne il suo utilizzo a livello clinico in sostituzione agli altri antibiotici della stessa classe per la maggior tolleranza da parte dell'organismo umano.

\section{1 \\ EPIDEMIOLOGIA MOLECOLARE DELLE INFEZIONI GENITALI DA C.TRACHOMATIS A BOLOGNA}

Pignanelli S.', Donati M. ', D’Antuono A. ${ }^{2}$, Shurdhi A.', Di Francesco A. ${ }^{3}$, Baldelli R. ${ }^{3}$, Burtica E. C. ${ }^{2}$, Della Bella E. ', Cevenini R.

'DMCSS - Sezione di Microbiologia

- Università degli Studi di Bologna, via Massarenti 9, 40138 Bologna ${ }^{2}$ DMCSS - Sezione di Dermatologia

- Università degli Studi di Bologna, via Massarenti 9, 40138 Bologna 3Dipartimento di Sanità Pubblica Veterinaria e Patologia Animale

- Università degli Studi di Bologna - via Tolara di Sopra 50,

40064 Ozzano Emilia (BO)

Introduzione. Chlamydia trachomatis rappresenta, nei Paesi industrializzati, una delle più frequenti cause di infezioni batteriche sessualmente trasmesse e costituisce un grave problema per la salute pubblica, per il rischio di complicanze cliniche (infertilità). Scopo del presente lavoro è stato quello di identificare, mediante sequenziamento genico, i sierotipi di $C$. trachomatis più frequentemente responsabili di infezioni genitali.

Metodi. Nel periodo compreso tra settembre 2005-maggio 2007 sono stati studiati 1.599 pazienti afferenti all'ambulatorio MTS del Policlinico S. Orsola-Malpighi di Bologna, per sospetta infezione genitale da C. trachomatis. La diagnosi di infezione è stata effettuata mediante isolamento di C. trachomatis in colture cellulari. I ceppi isolati sono stati successivamente tipizzati attraverso sequenziamento genico.

Risultati. La prevalenza dell'infezione genitale da C. trachomatis è risultata del 9.5\%, con una distribuzione maggiore nei pazienti di sesso maschile stranieri (22.2\%), rispetto a quelli italiani (14.3\%), e simile nelle pazienti di sesso femminile straniere (4.9\%) ed italiane (4.4\%). Il range di età maggiormente interessato dall'infezione è risultato compreso tra 15-24 anni nel sesso femminile e 2534 anni in quello maschile. Clinicamente sono risultati sintomatici il 25\% delle donne e l' $83.7 \%$ degli uomini. I sierotipi $G, E$ ed $F$ sono risultati i più diffusi nella nostra popolazione.

Conclusioni. I dati ottenuti concordano con quelli della letteratura riguardo a frequenza e distribuzione dell'infezione da C. trachomatis. La tipizzazione dei sierotipi isolati ha evidenziato differenze, in termini di prevalenza, rispetto ai sierotipi isolati nella stessa area geografica negli anni ' 90 . Mentre in quel periodo E (36\%) e D (26\%) risultavano i sierotipi più diffusi, rispetto a $\mathrm{G}(10.5 \%), \mathrm{K}$ (10.5\%), F (5.6\%), J (5.6\%), H (5.6\%) ed I (1\%), oggi G $(23 \%)$, E (20\%) ed $\mathrm{F}(20 \%)$ si sono dimostrati più frequenti di D (13\%), J (10\%), K (6\%), H (3\%) ed I (3\%). Tali modificazioni sono presumibilmente correlabili al notevole aumento dei flussi migratori. 